\title{
Epidermal growth factor receptor and activated epidermal growth factor receptor expression in gastrointestinal carcinoids and pancreatic endocrine carcinomas
}

\author{
Bettina Papouchado ${ }^{1}$, Lori A Erickson ${ }^{1}$, Audrey L Rohlinger ${ }^{2}$, Timothy J Hobday ${ }^{3}$, \\ Charles Erlichman ${ }^{3}$, Matthew M Ames ${ }^{4}$ and Ricardo V Lloyd ${ }^{1}$ \\ ${ }^{1}$ Department of Laboratory Medicine and Pathology, Mayo Clinic College of Medicine, Rochester, MN 55905, \\ USA $;{ }^{2}$ Department of Biostatistics, Mayo Clinic College of Medicine, Rochester, MN 55905, USA; ${ }^{3}$ Department \\ of Medical Oncology, Mayo Clinic College of Medicine, Rochester, MN 55905, USA and ${ }^{4}$ Department of \\ Pharmacology, Mayo Clinic College of Medicine, Rochester, MN 55905, USA
}

\begin{abstract}
The epidermal growth factor receptor (EGFR) plays an important role in the pathogenesis of many tumors. To analyze the expression of EGFR and activated EGFR in well-differentiated neuroendocrine carcinomas including primary and metastatic gastrointestinal carcinoid tumors and pancreatic endocrine tumors (PET), we examined 58 gastrointestinal carcinoid tumors and 48 PET using immunohistochemistry, Western blotting, and RT-PCR. EGFR and activated EGFR (P-EGFR) were expressed by both gastrointestinal carcinoids and PET in primary and metastatic tumors, although a higher percentage of gastrointestinal carcinoid tumors expressed EGFR and activated EGFR. Western blotting detected a $170 \mathrm{kDa}$ band for both EGFR and activated EGFR in three primary carcinoid tumors and two metastatic carcinoid tumors to the liver. RT-PCR analysis confirmed the expression of EGFR mRNA in both primary and metastatic carcinoid tumors. Patients with activated EGFR expression in their primary PET had a significantly worse prognosis compared to those who did not express activated-EGFR $(P=0.043)$. These results indicate that gastrointestinal carcinoid tumors as well as PET express EGFR and activated EGFR, and that expression is more common in gastrointestinal carcinoid tumors compared to pancreatic endocrine tumors. These findings implicate the EGFR and P-EGFR signal transduction pathway in the pathogenesis of these neuroendocrine tumors and suggest that targeted therapy directed against the EGFR tyrosine kinase domain may be a useful therapeutic approach in patients with unresectable metastatic gastrointestinal carcinoid tumors and pancreatic endocrine tumors.

Modern Pathology (2005) 18, 1329-1335. doi:10.1038/modpathol.3800427; published online 6 May 2005
\end{abstract}

Keywords: carcinoid; pancreatic endocrine tumor, EGFR, P-EGFR

Gastrointestinal carcinoid tumors and pancreatic endocrine tumors (PET) have common histopathologic characteristics such as production of chromogranin and synaptophysin. ${ }^{1}$ The tumors are also characterized by slow growth. When they become malignant, these tumors frequently metastasize to the liver and lymph nodes and are classified as welldifferentiated neuroendocrine carcinomas. Almost half of the metastatic neuroendocrine carcinoid tumors secrete excessive amounts of biogenic

Correspondence: Dr RV Lloyd, MD, PhD, Department of Laboratory Medicine and Pathology, Mayo Clinic, 200 First Street SW, Rochester, MN 55905, USA.

E-mail: lloyd.ricardo@mayo.edu

Received 3 January 2005; revised 15 March 2005; accepted 17 March 2005; published online 6 May 2005 amines and/or neuropeptides, causing a characteristic hypersecretion syndrome. Somatostatin analogues effectively palliate clinical syndromes due to hormone production and can cause rare regression or stabilization of tumor growth. The benefits of other systemic therapy such as interferon and chemotherapy is limited.

Recent studies have shown that the epidermal growth factor receptor (EGFR) plays a critical role in cancer development and progression, including cell proliferation, apoptosis, angiogenesis, and metastatic spread. ${ }^{2}$ EGFR is part of family of four closely related receptors: EGFR (ErbB-1), HER 2/neu (ErbB2), HER-3 (ErbB-3), and HER-4 (Erb-4). ${ }^{2}$ After ligand binding, EGFR dimerization with itself or other members of the HER family leads to high-affinity ligand binding, activation of the intrinsic protein 
tyrosine kinase activity, and tyrosine autophosphorylation. These events lead to activation of a cascade of biochemical and physiological responses that are involved in receptor-mediated signal transduction, cell mitogenesis and cell transformation., ${ }^{2,3}$ Many epithelial tumors and tumor-derived cell lines have been found to overexpress EGFR. Various studies have shown that EGFR is expressed in carcinoids and pancreatic endocrine tumors. ${ }^{4-8}$ However, the activated or phosphorylated form of EGFR (P-EGFR) has been examined in only a few neuroendocrine tumors, ${ }^{9}$ and it has not been studied in primary gastrointestinal carcinoid or pancreatic endocrine tumors.

Since EGFR overexpression has been correlated with disease stage, reduced survival and development of tumor metastases in a number of malignancies, ${ }^{6,10-13}$ a variety of different approaches are currently being used to treat patients with malignant tumors which express EGFR including targeting EGFR with anti-EGFR monoclonal antibodies and tyrosine kinase small molecule inhibitors to prevent autophosphorylation and downstream intracellular signaling. ${ }^{10,11}$

To evaluate the role of EGFR and P-EGFR expression in gastrointestinal neuroendocrine tumors, we studied the expression of this receptor by immunohistochemistry in primary and metastatic gastrointestinal neuroendocrine tumors. EGFR protein and mRNA expression were also analyzed by Western blotting and RT-PCR, respectively. The major aims of this study were: (a) to determine and compare EGFR and P-EGFR expression in human gastrointestinal carcinoid and pancreatic neuroendocrine carcinoma in primary and metastatic tumors; and (b) to correlate the extent of expression with clinicopathological parameters.

\section{Materials and methods}

\section{Patient Selection}

In all, 58 cases of gastrointestinal carcinoids from the small bowel and 48 cases of PET diagnosed between 1910 and 2003 were retrieved from the surgical pathology files of the Mayo Clinic (Rochester, MN, USA) for the present study. The material comprises primary tumors and matching metastases. The study was approved by Institutional Review Board at Mayo Clinic.

\section{Immunohistochemistry}

Polyclonal rabbit antibody to C-terminus epitope of EGFR were obtained from Santa Cruz Biotechnology, Santa Cruz, CA, USA (cat \#1005; dilution 1:250) and mouse monoclonal antibody to P-EGFR (Tyr1068) from Cell Signaling Technology, Beverly, MA, USA (cat \#2236S; dilution 1:100). ${ }^{9}$ Immunostaining for insulin, glucagon, gastrin and somatostatin was performed as previously reported. ${ }^{14}$ Tissue sections were microwave heated for $5 \mathrm{~min}$ in an $800-\mathrm{W}$ oven in citrate buffer $(0.1 \mathrm{mM}, \mathrm{pH} 6.0)$. Sections were then incubated at room temperature overnight with primary antibody. Immunostaining was performed with the avidin-biotin complex (ABC) method (Vector, Burlingame, CA, USA). Negative controls consisted of substituting normal serum for primary antibodies.

Immunoreactivity was qualitatively scored from 0 to $3+(0=$ negative; $1+=$ weak staining in $>10 \%$ of tumor cells; $2+=$ moderate staining in $>10 \%$; and $3+=$ strong staining in $>10 \%$ of tumor cells). Tissues were graded positive for EGFR or P-EGFR if definite staining was present in $>10 \%$ of the cells. Paraffin blocks from the A431 vulvar squamous cell carcinoma cell lines was used as positive controls for EGFR and P-EGFR.

\section{Western Blotting}

Western blotting was performed as previously described $^{15}$ using fresh frozen tissues from three primary ileal carcinoids and two liver metastases of ileal carcinoids. Briefly, proteins were extracted in the presence of protease inhibitors. One-dimensional sodium dodecyl sulfate polyacrylamide gel electrophoresis was then performed on a $12 \%$ gel. The electrophoresed protein $(50 \mu \mathrm{g})$ was transformed to a polyvinylidene difluoride membrane (Bio-Rad, Richmond, CA, USA) and subjected to immunoblot analysis with antibodies directed against EGFR (1:200) and P-EGFR (1:1000). The reaction product was detected by enhanced chemiluminescence (Amersham, Arlington Heights, IL, USA).

\section{RT-PCR}

Total RNA extraction was performed using the TRIzol reagent kit (Life Technologies) using fresh frozen tissues from three primary ileal carcinoids and two liver metastases of ileal carcinoids. Firststrand complementary DNA (cDNA) was prepared from total RNA by using a first-strand RT-PCR kit (Stratagene, La Jolla, CA, USA). The RT reaction was performed at $42^{\circ} \mathrm{C}$ for $60 \mathrm{~min}$ in a final volume of $50 \mu \mathrm{l}$ containing $1 \mu \mathrm{g}$ of total RNA. The reaction product was then heated at $95^{\circ} \mathrm{C}$ for $5 \mathrm{~min}$ and immediately placed on ice.

The oligonucleotide primers for EGFR were as follows: sense, 5'-TCT CAG CAA CAT GTC GAT GG-3'; antisense, $5^{\prime}$-TCG CAC TTC TTA CAC TTG CC- $3^{\prime}$. Spanning four introns between exons 8 and 12 of the human EGFR genomic DNA (\#X00588), these primers generate a 473-bp product (702-1175). The integrity of the RNA from each sample was verified by RT-PCR for hypoxanthine phosphoribosyltransferase (HPRT). The primers used to identify HPRT (\#M31642) were as follows: sense, 5'-CTT GCT CGA GAT GTG ATG AGG-3'; antisense, 5' -GTC TGC ATT GTT TTG CCA GTG $-3^{\prime}$. These primers 
span three introns between exons 3 and 6 and generate a 290-bp product (230-519). The PCR was performed in $25 \mu$ l final reaction volumes containing the following: $2.5 \mu \mathrm{l}$ of $\mathrm{RT}$ reaction product as template DNA, $1 \times$ PCR buffer (Promega, Madison, WI, USA), $2 \mu \mathrm{mol} / \mathrm{l} \mathrm{MgCl}_{2}, 0.2 \mu \mathrm{mol} / \mathrm{l}$ of each deoxynucleotide (Roche Molecular Biochemicals, Indianapolis, IN, USA), $0.2 \mu \mathrm{M}$ of sense and antisense primers for EGFR, and $1.25 \mathrm{U}$ Taq DNA polymerase (Promega). Programmable temperature cycling (GeneAmp PCR System 9700, Applied Biosystems) was performed with the following cycle profile: $95^{\circ} \mathrm{C}$ for $5 \mathrm{~min}$, followed by $94^{\circ} \mathrm{C}$ for $30 \mathrm{~s}$, $61^{\circ} \mathrm{C}$ for $30 \mathrm{~s}$, and $72^{\circ} \mathrm{C}$ for $45 \mathrm{~s}$ (34 cycles) for EGFR and $94^{\circ} \mathrm{C}$ for $30 \mathrm{~s}, 58^{\circ} \mathrm{C}$ for $30 \mathrm{~s}$, and $72^{\circ} \mathrm{C}$ for $30 \mathrm{~s}(30$ cycles) for HPRT. After the last cycle, the elongation step was extended at $72{ }^{\circ} \mathrm{C}$ for $10 \mathrm{~min}$. A $20 \mu \mathrm{l}$ aliquot of PCR product was analyzed by gel electrophoresis using a $2 \%$ agarose gel and was stained with ethidium bromide. A $100 \mathrm{bp}$ ladder (Roche) was used as the standard.

\section{Statistical Methods}

$\chi^{2}$ or Fisher's exact tests were used to compare EGFR and P-EGFR expression between primary and metastatic tumors within each tumor type. Cancerspecific survival was determined by the KaplanMeier method. There were no deaths from causes other than pancreas or small bowel neuroendocrine tumors. The associations of EGFR and P-EGFR expression with death from disease were assessed using log rank tests and Cox proportional hazards regression models and summarized with hazard ratios and 95\% confidence intervals (CI). Comparisons of features between the two tumor types were evaluated using $\chi^{2}$, Fisher's exact, and Wilcoxon rank sum tests. Statistical analyses were performed using the SAS software package (SAS Institute; Cary, NC, USA) and $P$-values $<0.05$ were considered statistically significant.

\section{Results}

\section{Clinicopathologic Findings}

In all, 58 gastrointestinal carcinoids and 48 PET were analyzed. Only patients who had metastatic disease at the time of surgery were selected for the present study. With the exception of three cases, two of which occurred in the duodenum and one in the jejunum, all gastrointestinal carcinoid tumors originated in the ileum. The sites of metastatic disease were mainly liver (45 of 58 gastrointestinal carcinoids, and 29 of 48 PET) and lymph node (33 of 58 gastrointestinal carcinoids, and 22 of 48 PET). All patients with carcinoid tumors with liver metastases had the carcinoid syndrome $(n=45)$ and 10 patients had carcinoid heart disease. Forty of the PET were studied by immunostaining and showed seven insulinomas, seven gastrinomas, two somatostatino- mas and two glucagonomas. There were 22 nonfunctional tumors in the PET group.

\section{Expression of EGFR and P-EGFR by Immunohistochemistry}

Immunohistochemical study showed mainly cytoplasmic staining and some weak membrane staining for EGFR in the primary and metastatic carcinoid and PET. Activated EGFR immunostaining was usually distributed focally and was localized mainly in the cytoplasm of tumor cells (Figure 1 and Table 1).

Expression of EGFR by immunohistochemistry was present in $91 \%$ of primary gastrointestinal carcinoids and $98 \%$ of metastatic deposits. In contrast, EGFR expression was significantly lower in both primary and metastatic PET $(P$-value $<0.01)$. Only $25 \%$ and $18 \%$ of primary and metastatic PET showed EGFR expression, respectively (Table 1). Similar pattern of expression was observed for P-EGFR. P-EGFR expression was present in 54 and $42 \%$ of primary and metastatic gastrointestinal carcinoids, respectively. The expression was significantly lower in primary PET. P-EGFR was detected in only $23 \%$ of primary PET $(P$-value $<0.001)$; and $32 \%$ of metastatic PET $(P$-value $=0.337)$ (Table 2$)$.

\section{Western Blotting and RT-PCR}

Western blotting showed a $170 \mathrm{kDa}$ band for both EGFR and P-EGFR in three primary and two metastatic carcinoid tumors (Figure 2). The intensity of the band was greater in the positive control sample (A431 cell line) compared to the carcinoids suggesting lower levels of EGFR in carcinoid tumors.

RT-PCR analysis showed a 473 bp transcript in the three primary and two metastatic carcinoid for EGFR supporting the immunohistochemical and Western blotting data (Figure 3).

\section{Association between P-EGFR Expression and EGFR}

A significant relationship was observed between P-EGFR and EGFR in patients with primary gastrointestinal carcinoids and PET. P-EGFR was found in $60 \%$ (31 of 52) of EGFR-positive primary gastrointestinal carcinoids compared with 0 of 5 EGFR-negative primary gastrointestinal carcinoids $(P$-value $=0.016)$ and $50 \%$ (six of 12$)$ of EGFRpositive primary PET compared with 14\% (five of 34) of EGFR-negative primary gastrointestinal carcinoids ( $P$-value $=0.020)$ (data not shown).

\section{Primary and Metastatic Tumor Expression of EGFR and P-EGFR}

There was a significant association between primary and metastatic EGFR expression for patients with 

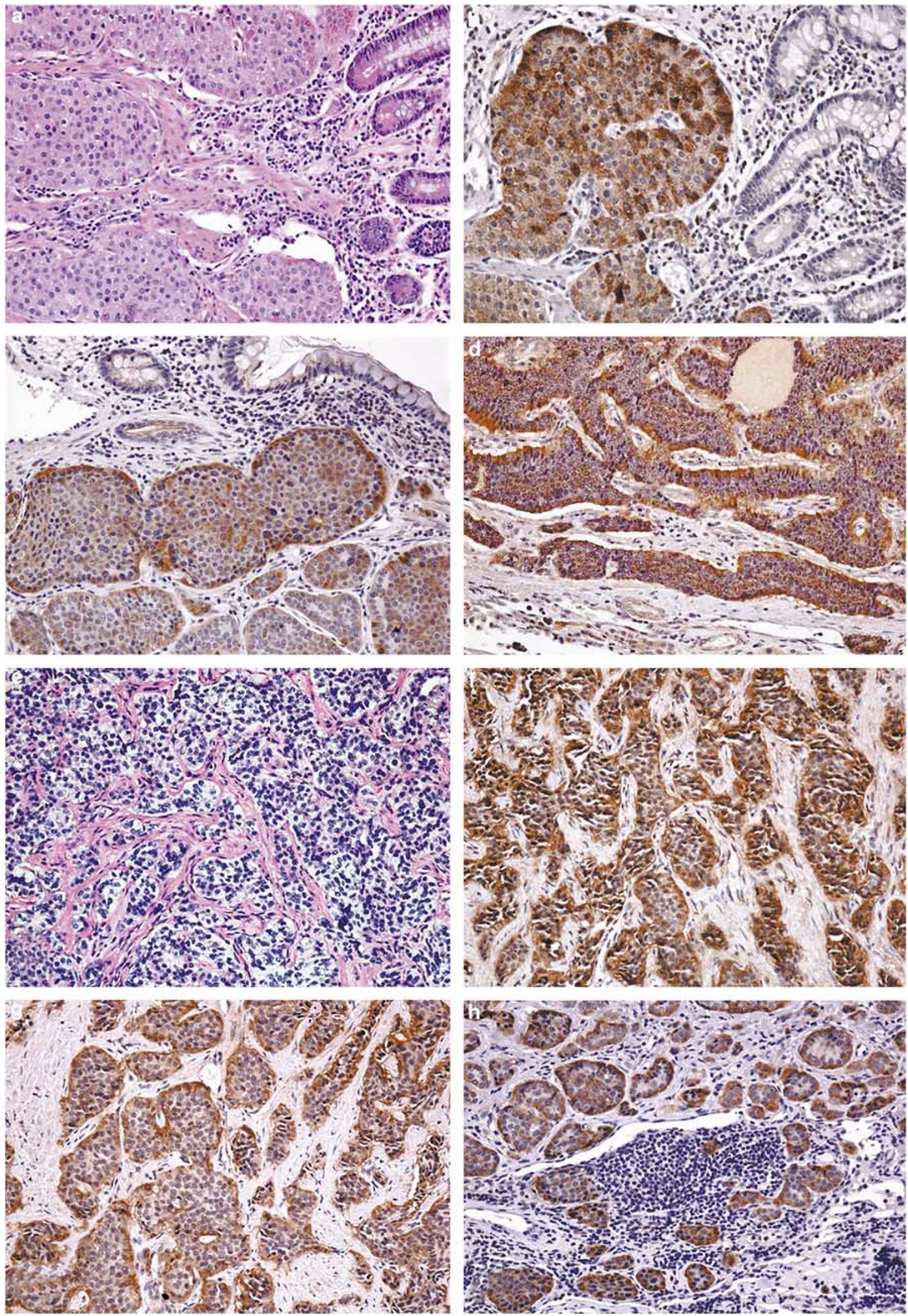
Table 1 Immunohistochemical expression of EGFR in primary and metastatic neuroendocrine tumors

\begin{tabular}{lcc}
\hline & $\begin{array}{c}\text { Small bowel } \\
(\mathrm{n}=58)\end{array}$ & $\begin{array}{c}\text { Pancreas } \\
(\mathrm{n}=48)\end{array}$ \\
\hline $\begin{array}{c}\text { EGFR-primary } \\
\text { Positive } \\
\text { Negative }\end{array}$ & $91.2 \%(52 / 59)$ & $25.0 \%(12 / 48)$ \\
& $8.8 \%(5 / 57)$ & $75.0 \%(36 / 48)$ \\
EGFR-metastasis & \multicolumn{2}{c}{$P<0.01$} \\
$\quad$ Positive & $98.1 \%(51 / 52)$ & $17.5 \%(7 / 40)$ \\
$\quad$ Negative & $1.9 \%(1 / 52)$ & $82.5 \%(33 / 40)$ \\
& \multicolumn{2}{c}{$P<0.01$} \\
& \multicolumn{2}{c}{}
\end{tabular}

Table 2 Immunohistochemical expression of P-EGFR in primary and metastatic neuroendocrine tumors

\begin{tabular}{|c|c|c|}
\hline & $\begin{array}{l}\text { Small bowel } \\
\quad(\mathrm{n}=58)\end{array}$ & $\begin{array}{c}\text { Pancreas } \\
(\mathrm{n}=48)\end{array}$ \\
\hline \multicolumn{3}{|c|}{ P-EGFR-primary } \\
\hline Positive & $54.4 \%(31 / 57)$ & $23.4 \%(11 / 47)$ \\
\hline \multirow{2}{*}{ Negative } & $45.6 \%(26 / 57)$ & $76.6 \%(36 / 47)$ \\
\hline & \multicolumn{2}{|c|}{$P<0.01$} \\
\hline \multicolumn{3}{|c|}{$P$-EGFR metastasis } \\
\hline Positive & $42.3 \%(22 / 52)$ & $32.5 \%(13 / 40)$ \\
\hline \multirow{2}{*}{ Negative } & $57.7 \%(30 / 52)$ & $67.5 \%(27 / 40)$ \\
\hline & \multicolumn{2}{|c|}{$P<0.344$} \\
\hline
\end{tabular}

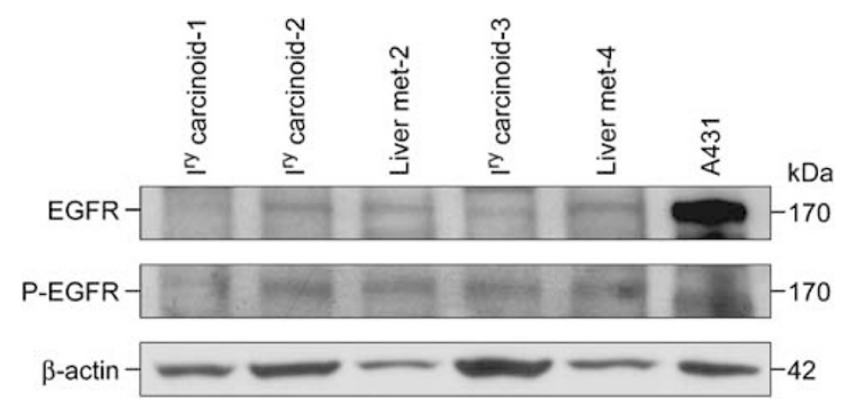

Figure 2 Western blot analysis of EGFR top and P-EGFR expression in three primary and two metastatic small intestinal carcinoid tumors. In all, $50 \mu \mathrm{g}$ of protein was loaded in each lane and stained with antibodies against EGFR and P-EGFR and electrophoresed on a polyacrylamide gel, transformed to a membrane. The final results were visualized by enhanced chemiluminescence. A $170 \mathrm{kDa}$ band was detected for both EGFR and P-EGFR. A vulvar squamous cell carcinoma cell line (A431) was used as a positive control.

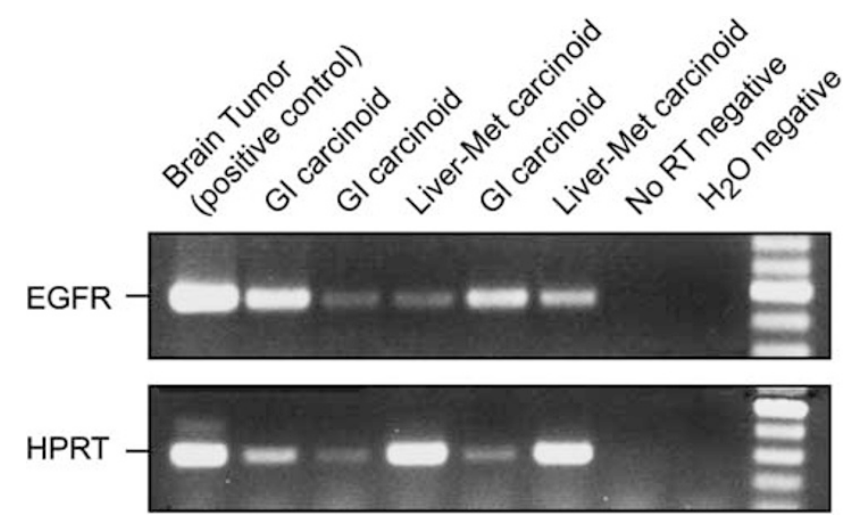

Figure 3 RT-PCR analysis of three primary and two metastatic small intestinal carcinoid tumors. In all, $5 \mu \mathrm{g}$ of total RNA was converted to cDNA by reverse transcriptase and the cDNA was amplified with specific primers. The product was separated on an agarose gel and a $473 \mathrm{bp}$ band was detected after ethidium bromide staining. A brain tumor (glioblastoma multiforme)expressing EGFR was used as a positive control.

PET $(P$-value $=0.034)$. More specifically, if the patient had positive EGFR expression in the primary tumor, they were more likely to have a positive EGFR expression in the metastatic tumor. There was not a statistically significant association between primary and metastatic EGFR expression among patients with small bowel tumors; however, most of these specimens had positive EGFR expression. There was also a significant association between primary and metastatic P-EGFR expression for patients with gastrointestinal carcinoids $(P$-value $<0.001$ ). If the patient had positive P-EGFR expression in the primary, they were more likely to have positive P-EGFR expression in the metastatic tumor (data not shown).

\section{Hormone and P-EGFR Expression}

Comparison of P-EGFR immunostaining with hormone production did not show a correlation with the production of specific hormones including insulin and gastrin. There was no relationship between the 10 patients with carcinoid heart disease and EGFR or P-EGFR expression.

\section{Survival by EGFR and P-EGFR Expression in Primary and Metastatic Gastrointestinal Carcinoids and PET}

Survival analyses showed a significant association between P-EGFR expression in primary PET

Figure 1 (a) Hematoxylin and eosin sections of a primary small intestinal carcinoid tumor involving the mucosa, submucosa, and muscularis propria. (b) Immunohistochemical staining for EGFR in a small intestinal carcinoid shows strong $(3+)$ staining of the tumor cells. (c) Immunohistochemical stains for P-EGFR in a small intestinal carcinoid showing focal but strong (3+) staining. (d) Metastatic carcinoid tumor to the liver showing strong immunostaining for EGFR $(3+)$ in most of the tumor cells. (e) Primary pancreatic endocrine tumor staining with hematoxylin and eosin. The tumor cells are arranged in nests in a desmoplastic background. (f) Immunohistochemical staining for EGFR in a primary pancreatic endocrine tumor showing diffuse strong $(3+)$ staining in the tumor cells. (g) Immunohistochemical staining for P-EGFR in a primary pancreatic endocrine tumor showing patchy staining for P-EGFR. (h) Metastatic pancreatic endocrine tumor to a lymph node showing strong $(3+)$ immunostaining for P-EGFR in most of the tumor cells. 


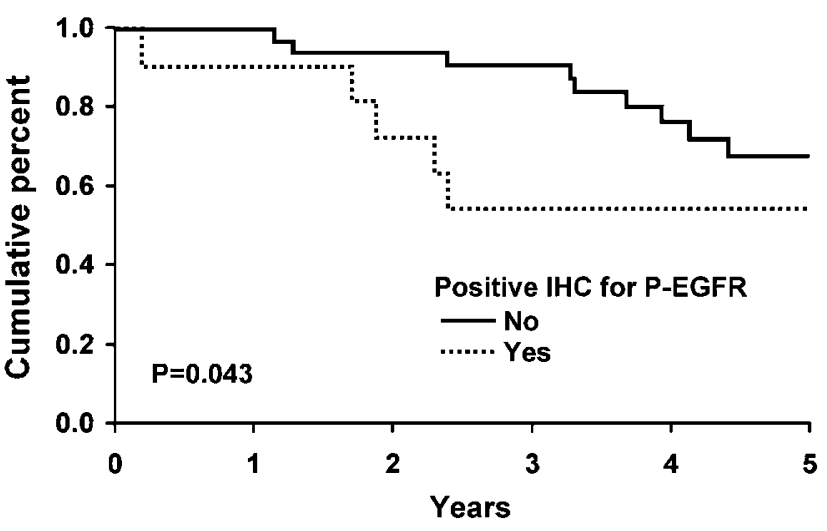

Figure 4 Survival analysis stratified by expression of activated EGFR (P-EGFR) plotted as the proportion of patients surviving from the date of surgery vs time in years using the Kaplan-Meier method $(P=0.043$ by log rank test).

$(P=0.043$; hazard ratio $=2.65 ;$ CI $(1.03,6: 78)$, indicating that they were 2.65 times more likely to die from disease than patients whose tumors were negative for P-EGFR (Figure 4). No other associations were statistically significant.

\section{Discussion}

We examined EGFR and P-EGFR expression in primary gastrointestinal carcinoid and pancreatic endocrine tumors and their metastasis. These analyses showed a significant difference in the expression of EGFR and P-EGFR between these two neuroendocrine tumor types with higher levels in the primary and metastatic gastrointestinal carcinoids compared to the pancreatic endocrine tumors.

Although earlier studies examined EGFR expression in carcinoids and pancreatic endocrine tumors ${ }^{4-8}$ this study is the first to examine activated or P-EGFR in these tumors. Western blot analysis supported the immunohistochemical observations of EGFR and P-EGFR expression by the detection of a $170 \mathrm{kDa}$ protein band in primary and metastatic carcinoid tumors. RT-PCR analysis of these same tumors showed EGFR transcripts for EGFR in primary and metastatic tumors, although the level of the transcript was relatively less than in the positive control glioblastoma multiforme tumor.

Although small bowel and pancreatic endocrine tumors have similar histopathologic features, both groups of tumors express neuroendocrine markers such as chromogranin and synaptophysin. ${ }^{1}$ Our study indicates that biologically, these tumors may be different based on the expression of growth factor receptors expressed by the tumors such as EGFR and P-EGFR in this study. Srivastava et $a l^{8}$ had a higher percentage of PET positive for EGFR (65\%) than in the current series $(25 \%)$. These differences may be related to different antibodies used, differences in the percentage of malignant cases studied $(100 \%$ in the current series) as well as other variables. Other recent studies suggest that there are also molecular differences between these two groups of neuroendocrine tumors. ${ }^{16-20}$

In a study of 20 pancreatic endocrine tumors using loss of heterozygosity (LOH), Beghelli, et al, ${ }^{20}$ reported frequent $\mathrm{LOH}$ on chromosome $17 \mathrm{p} 13$ which was associated with extrapancreatic extension of disease. Other investigators have reported $\mathrm{LOH}$ as a frequent occurrence in PET but not in midgut carcinoids. ${ }^{16} \mathrm{~A}$ recent analysis of $\mathrm{CpG}$ island methylation showed that the methylation profile of carcinoid tumors was quite different from that of PET. ${ }^{16}$

The role of EGFR in the growth of carcinoid and PET is uncertain. Our study suggests that P-EGFR expression in PET is also a poor prognostic sign. Studies with other tumor types such as esophageal carcinoma also suggested that patients who had tumors overexpressing EGFR were associated with a worse prognosis. ${ }^{21,22}$ EGFR expression in other neuroendocrine tumors such as pituitary tumors has been associated with more aggressive tumors. ${ }^{23,24}$

In a recent study of neuroendocrine gastrointestinal tumor cell lines, Hopfner et al, ${ }^{11}$ used an inhibitor of EGFR-sensitive tyrosine kinase to suppress growth of these cell lines and induction of apoptosis and cell-cycle arrests in a human insulinoma (CM) and a human pancreatic carcinoid (BON) cell lines. These findings indicate that EGFR was present and active in these cells and could be regulated by targeting the tyrosine kinase receptor. These data taken together without findings that PEGFR is associated with a poor prognosis at least in PET, suggests that targeting EGFR may be a therapeutic target to explore. We are conducting a phase II trial of gefitinib, a small molecule inhibitor of the EGFR tyrosine kinase domain, in carcinoids and PET through a multicenter phase II consortium.

In summary, analysis of EGFR and P-EGFR expression in gastrointestinal carcinoids and PET showed a higher percentage of cases of primary and metastatic gastrointestinal carcinoids expressing EGFR and P-EGFR compared to PET implicating the EGFR signal transduction pathway in the development of these tumors. These results suggest that targeted therapy directed against the EGFR tyrosine kinase domain may be a useful therapeutic approach.

\section{Acknowledgements}

We thank Shuya Zhang for technical assistance. This work was supported in part by grant from the Verto Institute.

\section{References}

1 Lloyd RV. The neuroendocrine and paracrine systems. In: Mills SE, Cartey D, Greenson JK, Oberman HA, Reuter V, Slater MH (eds). Sternberg's Diagnostic Surgical Pathology, 4th edn. Lippincott, Williams and Wilkins: Philadelphia, 2004, pp 507-519. 
2 Riese II DJ, Stern DF. Specificity within the EGF family/ErbB receptor family signaling network. Bioessays 1998;20:41-48.

3 Baselga J. Why the epidermal growth factor receptor? The rationale for cancer therapy. Oncologist 2002; 7(Suppl 4):2-8.

4 Oberg K. Expression of growth factors and their receptors in neuroendocrine gut and pancreatic tumors, and prognostic factors for survival. Ann NY Acad Sci 1994;733:46-55.

5 Nilsson O, Wangberg B, Kolby L, et al. Expression of transforming growth factor alpha and its receptor in human neuroendocrine tumours. Int J Cancer 1995;60: 645-651.

6 Peghini PL, Iwamoto M, Raffeld M, et al. Overexpression of epidermal growth factor and hepatocyte growth factor receptors in a proportion of gastrinomas correlates with aggressive growth and lower curability. Clin Cancer Res 2002;8:2273-2285.

7 Krishnamurthy S, Dayal Y. Immunohistochemical expression of transforming growth factor alpha and epidermal growth factor receptor in gastrointestinal carcinoids. Am J Surg Pathol 1997;21:327-333.

8 Srivastava A, Alexander J, Lomakin I, et al. Immunohistochemical expression of transforming growth factor alpha and epidermal growth factor receptor in pancreatic endocrine tumors. Hum Pathol 2001;32: 1184-1189.

9 Onguru O, Scheithauer BW, Kovacs K, et al. Analysis of epidermal growth factor receptor and activated epidermal growth factor receptor expression in pituitary adenomas and carcinomas. Mod Pathol 2004;17: 772-780.

10 Grunwald V, Hidalgo M. Developing inhibitors of the epidermal growth factor receptor for cancer treatment. J Natl Cancer Inst 2003;95:851-867.

11 Hopfner M, Sutter AP, Gerst B, et al. A novel approach in the treatment of neuroendocrine gastrointestinal tumours. Targeting the epidermal growth factor receptor by gefitinib (ZD1839). Br J Cancer 2003;89: 1766-1775.

$12 \mathrm{Di}$ Lorenzo G, Tortora G, D’Armiento FP, et al. Expression of epidermal growth factor receptor correlates with disease relapse and progression to androgenindependence in human prostate cancer. Clin Cancer Res 2002;8:3438-3444.
13 Ciardiello F, Tortora G. A novel approach in the treatment of cancer: targeting the epidermal growth factor receptor. Clin Cancer Res 2001;7:2958-2970.

14 Chang HF, Batts KP, Lloyd RV, et al. Progressive significance of p27, ki-67 and topoisomerase II expression in clinically nonfunctioning pancreatic endocrine tumors. Endocr Pathol 2000;11:229-241.

15 Riss D, Jin L, Qian X, et al. Differential expression of galectin-3 in pituitary tumors. Cancer Res 2003;63: 2251-2255.

16 Chan AO, Kim SG, Bedeir A, et al. CpG island methylation in carcinoid and pancreatic endocrine tumors. Oncogene 2003;22:924-934.

17 D’Adda T, Pizzi S, Azzoni C, et al. Different patterns of $11 \mathrm{q}$ allelic losses in digestive endocrine tumors. Hum Pathol 2002;33:322-329.

18 Chung DC, Smith AP, Louis DN, et al. A novel pancreatic endocrine tumor suppressor gene locus on chromosome $3 p$ with clinical prognostic implications. J Clin Invest 1997;100:404-410.

19 Chung DC, Brown SB, Graeme-Cook F, et al. Localization of putative tumor suppressor loci by genome-wide allelotyping in human pancreatic endocrine tumors. Cancer Res 1998;58:3706-3711.

20 Beghelli S, Pelosi G, Zamboni G, et al. Pancreatic endocrine tumours: evidence for a tumour suppressor pathogenesis and for a tumour suppressor gene on chromosome 17p. J Pathol 1998;186:41-50.

21 Itakura Y, Sasano H, Shiga C, et al. Epidermal growth factor receptor overexpression in esophageal carcinoma. An immunohistochemical study correlated with clinicopathologic findings and DNA amplification. Cancer 1994;74:795-804.

22 Gibson MK, Abraham SC, Wu TT, et al. Epidermal growth factor receptor, p53 mutation, and pathological response predict survival in patients with locally advanced esophageal cancer treated with preoperative chemoradiotherapy. Clin Cancer Res 2003;9:64616468.

23 LeRiche VK, Asa SL, Ezzat S. Epidermal growth factor and its receptor (EGF-R) in human pituitary adenomas: EGF-R correlates with tumor aggressiveness. J Clin Endocrinol Metab 1996;81:656-662.

24 Jaffrain-Rea ML, Petrangeli E, Lubrano C, et al. Epidermal growth factor binding sites in human pituitary macroadenomas. J Endocrinol 1998;158:425-433. 